\title{
DBT-based skills training for family members of suicidal patients - open clinical feasibility trial in a psychiatric outpatient context'
}

Mia Rajalin ( $\sim$ mia.rajalin@sll.se)

Umea Universitet Medicinska fakulteten https://orcid.org/0000-0001-6513-274X

\section{Tatja Hirvikoski}

Karolinska Institutet

\section{Ellinor Salander-Renberg}

Umea Universitet Medicinska fakulteten

Jussi Jokinen

Karolinska Institutet

\section{Research article}

Keywords: suicide attempt, suicide prevention, family psychoeducation, DBT skills training, Family Connections, burden

Posted Date: March 3rd, 2020

DOl: https://doi.org/10.21203/rs.3.rs-15801/v1

License: (9) (1) This work is licensed under a Creative Commons Attribution 4.0 International License. Read Full License 


\section{Abstract}

Background: The involvement of significant others is an objective in suicide prevention and there is a need for development of interventions for this group. The aim of this open naturalistic study was to evaluate the feasibility and the preliminary efficacy of Family Connections (FC) for significant others of suicidal patients, a nine-week manualized skills training program based on dialectical behavior therapy (DBT) in a clinical context.

Methods: The intervention program aims to enhance the knowledge of symptoms and behaviors connected with attempted suicide. Furthermore it introduces stress-coping strategies and emotion regulation skills. Out of 132 participants in total, 104 (79\%) completed the program. Included in the analysis were the 86 (65\%) participants who completed the program and also had both pre- and postintervention assessments. The Burden Assessment scale (BAS), the Questions About Family Members scale (QAFM), and the Five Facet Mindfulness Questionnaire (FFMQ), were used to assess perceived burden, relationship climate and five facets of mindfulness respectively.

Results: The results showed a significant reduction in perceived burden, measured with BAS and a significant change in the mindfulness scale FFMQ subscale Acting with Awareness, which indicates an enhanced ability to be present in the moment. None of the four subscales in QAFM showed significant changes between the pre- and post-intervention assessments.

Conclusions: The results support the feasibility and potential value of the implementation of an intervention for significant others of suicidal patients in psychiatric care. Randomized controlled studies are needed to generate further evidence.

\section{Background}

The suicidal act, even if not leading to death, has an immense impact not only on the individual performing it, but also on his or her environment. Being a very painful experience for everybody involved, the questions in the wake of a suicide or a suicide attempt often are "why?" or "what could I have done?". The wellbeing of close relatives and friends is strongly affected and they are themselves in an elevated risk of suicide $[1 ; 2]$. The need for evidence-based interventions aimed at next-of-kin is an important objective for modern public health care. The use of feasibility studies is one way of finding adapted interventions that can be both effective and efficacious [3].

Family psychoeducation (FPE) is a well-established psychosocial treatment for patients with psychosis [4], and a recommendation in guidelines for treatment of schizophrenic patients [5]. In addition to traditional psychoeducation it also often includes common cognitive behavioral therapeutic elements and coping skills introduction [5]. According to McFarlane's review, FPE reduces relapse with $50-60 \%$ compared to treatment as usual in patients with schizophrenia. Thus, the importance of family members and friends in the care for a patient with a psychiatric disorder seems to be recognized even though not yet enough prioritized. 
Attention Deficit Hyperactivity Disorder (ADHD) and borderline personality disorder (BPD) are other psychiatric disorders that are partly characterized by strained relationships. An effort has been made in recent years regarding interventions for family members of adults with ADHD and BPD [6; 7]. The intervention for relatives of persons with BPD, Family Connections (FC), includes skills training based on dialectical behavior therapy (DBT) [7; 8]. Ekdahl and colleagues [9] conducted a study in 2014 on a family intervention containing DBT skills training and found that the participants, parents of self-harming patients at a Swedish child and adolescent psychiatry unit, benefited from the program. Though suicidal behavior certainly does occur in this patient group, the interventions have not addressed the issue of suicidal behavior specifically. There are some preliminary results that support the feasibility and acceptability of DBT-based skills training across populations [10]. Magne-Ingvar and colleagues [11] have earlier pointed out the scarce existence of networks around suicidal patients. They found that only $13 \%$ of the suicide attempters had a well-functioning relationship and suggested an integration of the social network in psychiatric clinical care.

Family members of suicide attempters are burdened by their involvement in the patient. They worry not only about the patient, but also for themselves and their future [12]. In 2009 we conducted a pilot study evaluating an adapted version of FC for family members of suicidal patients [13]. The trial included thirteen relatives of suicide attempters, and after completing the program, the participants experienced a significant reduction in burden, an improved relation to the patient, and an increase in general well-being.

To our knowledge there are few studies on family skills training for relatives of suicidal patients. Sun and collaborators [14] evaluated a brief intervention for caregivers of suicidal people and found that a twohour education intervention improved family care-givers sense of ability to care for the suicidal patient and helped them to generate a more encouraging attitude in the relationship to the suicidal patient. In one study LeCloux and colleagues [15] conclude that the family network is crucial in suicide prevention and that there is a need for research to focus on implementation of interventions for this group.

\section{Family Psychoeducation}

As mentioned above, FPE has been shown to give benefits for patients and their families affected by the most severe psychiatric disorders. It is a method intended to impart knowledge of the illness in question, and to support the development of new strategies for coping and handling the problems that arise in a difficult situation. Family refers to anyone who is close to the patient; it does not have to be a relative. Psychological support is crucial to the quality of life, level of functioning, relapse rate and chance of recovery in both affected individuals and their families. This kind of support also increases families' satisfaction with the care provided [16].

Mindfulness and acceptance

Mindfulness has been included as a tool in various health and psychiatric settings over the past decades, owing to the benefits that have been demonstrated [17; 18]. The inclusion of mindfulness skills training in a family intervention alludes to reinforce acceptance without judgment, to allow a full experience of life. 
Further, it may also lead to awareness of one's inner values, and in the long run enhance the ability to self-regulate emotion. When reality is fully accepted as it is, the ability to discover and understand emotions, tolerate and allow them, whether they are enjoyable or not, is heightened. Mindfulness and acceptance have been shown to be effective in regulation of dysfunctional emotion, especially if combined with a non-judgmental attitude and validation [19; 20; 21].

Family Connections

The manualized family skills training program FC [7] is divided into six modules: psychoeducation, mindfulness, acceptance, emotion, validation and problem management. The program was adapted for family members and friends of suicidal patients [13] and a pilot study was performed in a specialist clinic, the Suicide Prevention Clinic at Karolinska University Hospital. There was preliminary support for the intervention; however, there is no knowledge about the feasibility in a wider clinical context. The demand for psychological support for family members of suicidal patients is obvious. Studies have described the heavy burden of having a relative with a severe psychiatric disorder and that the family members feel left out and sometimes even used by health care services [22; 23].

Aim of the study

The aim of this study was to further investigate the feasibility and preliminary efficacy of the adapted DBT-based skills training program Family Connections for significant others of suicidal patients, either in inpatient or outpatient care, in a psychiatric outpatient context in Sweden.

\section{Methods}

Study setting

The study was conducted within a public psychiatric service (Stockholm County Council) in the southern part of Stockholm, capital of Sweden, between the years 2008-2015. The skills training program was offered as an intervention to the next of kin of both in- and outpatients with suicidal behaviour, independent of the usual treatment. Suicidal behavior was defined as a range of suicidal behaviors, including serious suicidal ideation, planning or having attempted suicide. The Regional Ethical Review Board in Stockholm approved of the study protocols (Dnr 2013/840-31/3) and participants gave their written informed consent to participate in the research study.

\section{Participants}

The study included 132 relatives, partners or friends of suicidal patients in psychiatric care, 76 women $(58 \%)$ and 56 men (42\%). See table 2 for detailed information on types of relationships and other characteristics of the sample. During the time period 14 skills training groups were conducted. Group size varied between seven to fourteen people. Inclusion criteria were 18 years of age, knowledge in Swedish and having a close relationship to a suicidal patient within the clinic. 


\section{Enrollment}

Participants were informed of the skills training program through advertising at the psychiatric outpatients' services clinic, through information from healthcare professionals involved in the suicidal patient's treatment or in a few cases through recommendation from a friend or another contact within the healthcare system with knowledge of the program. The participants signed up for the study with an administrator and were given introductory information about the study. All participants, except for the first two groups, gave their written informed consent before startup and the completion of the pre-intervention assessments. Regarding the first two groups, the Regional Ethical Review Board approved of their inclusion in the study retrospectively. All participants in the first two groups gave their verbal consent for sharing information of the questionnaires.

The intervention

Family Connections for family members of suicidal patients was adapted and shortened by three sessions [13]. The program duration was 18 hours over nine weeks. Regarding the adaptation the main changes were conducted in the psychoeducational part. Latest research based information of risk factors for suicide attempts and completed suicide as well as information of psychiatric disorders with high suicide risk (mood disorders, personality disorders and substance abuse disorders) was introduced in the psychoeducation session. Further, information about recommended treatment options and a description of the suicidal process was given. Two psychologists trained in FC and DBT served as group leaders. The participants in all groups took part of the different educational steps in the same order and rate. Table 1 shows the content of the intervention.

\section{Assessments}

Feasibility

Criteria for being regarded as having completed the program was attending at least six sessions out of the nine given (67\%). The participants described their satisfaction with the program verbally in group and in an open questionnaire in the last session.

\section{Burden Assessment Scale (BAS)}

Burden Assessment Scale [24] was used to assess the participants' subjective experience of burden for being a relative to a suicidal person. The scale, consisting of 19 items, aims to measure both objective and subjective burdens of daily life in families with a member who has been seriously mentally ill during the last six month period. A high mean reflects higher burden in all subscales. BAS was validated in Sweden by Ivarsson and colleagues [25] and a factor analysis resulted in three factors: "disrupted activities", "feelings of guilt/worry", and "social strain". The scale has been shown to have excellent reliability, and ability to differentiate between family samples with different levels of burden and it is sensitive to changes over time [25; 24]. 
Questions About Family Members [26] is a self-rating instrument and aims to assess expressed emotion (EE) [27] in dyads. We used QAFM to explore the relationship between the participant and the patient from the participants' perspective. The scale consists of 30 items which are rated on a 5-point Likert scale with answers ranging from "almost always" to "almost never". QAFM has been homogenized by factor analysis, resulting in four subscales; two factors about "given" EE: critical comments and emotional overinvolvement and two factors about "perceived EE": perceived criticism and perceived emotional involvement. The score is summed for each subscale, and is divided by the sum of items in the subscale. A higher score reflects a more strained relationship in the three subscales Critical comments, Perceived criticism and Emotional over-involvement and thus are expected to decrease, whereas in the subscale Perceived emotional involvement, an increase is regarded positive for the relationship. The reliability in clinical and non-clinical groups is good (Cronbach's alpha is 0.73 for perceived criticism, 0.69 for perceived emotional involvement, 0.87 for critical comments and 0.81 for emotional over- involvement ) and there is acceptable predictive validity [26].

The Five Facet Mindfulness Questionnaire (FFMQ)

To assess mindfulness, the Five Facet Mindfulness Questionnaire was used [28]. The scale measures five facets of mindfulness: Observing, Describing, Acting with Awareness, Non-judging of Inner Experience, and Non-reactivity to Inner Experience. The scale consists of 39 statements and uses a 1-5 Likert scale ranging from "Never or very rarely true" to "Very often or always true". A high mean reflects a high level of the specific mindfulness facet. The subscales Acting with Awareness and Non-judging of inner experience are reversed, i.e. a high score reflects a low awareness regarding one's behavior and a high degree of self-judgement respectively. The FFMQ has been shown to have good psychometric properties with alpha coefficients adequate-to good (range .72 to .92). FFMQ was included in the fifth group and forward.

Statistical analyses

A missing values analysis was performed in SPSS [29] to detect systematic patterns of missing data. After that cases with more than $50 \%$ missing (i.e. no pre- or post-measurement) were excluded as well as cases who did not fulfill the criteria of participation; attendance of at least six out of nine sessions. In the next step a new analysis was performed to detect single cases of missing data. Since missing values were less than $5 \%$, an imputation was made. The method involved replacement of missing values based on the proportional answers of the whole sample for each item [30].

Initial analyses were carried out to evaluate skewness and kurtosis of the distributions with the Shapiro Wilk test. Data was cross-checked with both parametric and non-parametric (Wilcoxon Signed Ranks test for matched pairs) tests. Results did not diverge notably between parametric and non-parametric methods. Paired samples t-test was used to assess change from pre-test to post-test and these results are the ones reported. All tests were two-tailed (SPSS 23.0, 2015). The alpha value was set at $p<.05$. 
To calculate effect sizes, Cohen's $d$, we corrected for dependence among means in order to make direct comparisons to effect sizes from within-subjects studies [31]. Thus, we entered the correlations between the pre and post-intervention means in a regular online effect size calculator for within-subjects studies. To control for an overestimation of effect we also performed Rosenthal's $r$. Since the effect size $r$ did not alter the effect sizes, we report Cohen's d, small (0.2), medium (0.5) and large (0.8) [30].

\section{Results}

Demographic data and background characteristics

The total number of participants in this study was 132, whereas the final sample, included in the analyses, consists of 86 participants. Table 2 shows the characteristics of the participants. The distributions of these characteristics did not diverge in the sample of the 54 participants that completed the scale FFMQ.

Feasibility

Out of the total of 132 participants, 104 (79\%) completed the program, i.e. attended six or more sessions. The average number of attended sessions was eight. However, the criteria for being included in the analyses also commanded a completion of both pre- and post-intervention assessments, which 86 participants (65\%) fulfilled. Of the 86 participants included in the analysis, $41 \%$ completed all nine sessions.

Attrition

The relative distribution among men and women that dropped-out was equal, 27 (36\%) women and 19 (34\%) men, respectively. If the participant was living together with the patient there was a little less likelihood to drop out than if they did not live together, $66 \%$ versus $59 \%$. Regarding the relation to the patient parents $(71 \%)$, partners $(61 \%)$ and children ( $84 \%)$ completed the program to a higher extent compared with the groups of siblings and other relations. Figure 1 shows a flowchart of the sample.

Stated reasons for dropping out in an earlier stage were illness or other major life events (10 persons), lack of time because of work or studies (8 persons), wanting another type of intervention (3 persons). There were cancellations to the last session, and also drop-outs during the program where the reason for dropping out is not known (7 persons). Another reason for not being included in the final analyses was that either pre- or post-assessment was not filled in or that the questionnaire was incorrectly filled out.

Efficacy-related outcome measures

BAS

The change between the pre- and posttest on BAS was statistically significant in all subscales as well as in the total scale (table 3). The perceived Guilt/worry ( $p \otimes 0.000)$, the social strain ( $p \otimes 0.029)$ and the 
disruption in activities ( $p \bigotimes 0.016)$ as well as the perceived total burden ( $p \bigotimes 0.001$ ) were all reduced between the two measures. The effect size regarding this reduction was medium in the total and in the guilt/worry subscales. The effect size in the other two subscales was small. On an item-level, the change between pre- and post-intervention assessment was significant in three out of five items in the subscale Guilt/worry. Both guilt and worry were reduced after completion of the program.

FFMQ

The analysis showed a statistically significant change between pre- and post-intervention assessments in one of the five facets: Acting with Awareness, $p<0.006$ (table 3) which indicates an enhanced ability to be in the present moment. There was a statistical trend of gaining more skills in the facet Observing after completing Family Connections educational program, $p<0.056$. The effect size regarding this reduction was small. The facet Describing showed no significant change between pre- and post-intervention assessments as well as the facets Non-judging of Inner Experience and Non-reactivity to Inner Experience.

\section{QAFM}

None of the four subscales; perceived criticism (PC), perceived emotional involvement (PEI), critical comments (CC), and emotional over involvement (EOI) in QAFM showed significant changes between the pre- and post-intervention assessments (table 3). The participants' scores remained high in all four subscales. These values indicate a relationship under pressure.

\section{Discussion}

In this open feasibility study in a clinical psychiatric context, the manualized DBT-based skills training program FC for family members and friends of suicidal patients was evaluated. The aim was to gather more information about the feasibility and preliminary efficacy of the intervention in an outpatient psychiatric context. Earlier studies on FC have shown an increase in the wellbeing of family members and in the relationship to the patient $[33 ; 34 ; 7]$. In the pilot study we performed in a specialist clinic, the Suicide Prevention Clinic at the Karolinska University Hospital, similar results were found [13].

Out of the 132 initial participants 104 (79\%) completed the program. The results are based on the group of 86 participants who also completed pre and post intervention assessments (65\%). This percentage is in comparison with other studies of interventions for family members. A dropout rate of $20 \%$ is usually reported for educational groups for caregivers [34]. However, when program demands are higher, the dropout rate usually rises, too [35].

Wilks and colleagues [36] treated family members of persons with behavioral disorders in a six-month DBT-based intervention. They saw a reduction in stress level and an improvement in interpersonal measurements in the 20 participants (53\%) that completed the intervention. According to Wilks, the intervention did not require additional resources from the outpatient clinic; however, it entailed a demanding program regarding time consumption for the participants. 
Our shorter intervention had a higher degree of attendance and it can be noted that the participants in our study found the intervention interesting and the two-hour weekly session during nine weeks acceptable. In fact, all participants who completed the program (except for one), expressed satisfaction and appreciation with their participation. Participants especially mentioned the modules mindfulness and validation as the most valuable, which rhyme well with their assessments. The reduction of worry and guilt could partly be attributed to enhanced self-validation whereas the increased ability to act with awareness could be explained by implemented mindfulness skills. The opportunity of meeting others in a similar situation and to be able to share experiences was held as the most important feature of the program.

In comparison to other studies on caregivers to severely mentally ill patients [25; 24], the degree of participants' perceived burden was somewhat lower in our study at baseline. Compared to a chronic psychiatric condition, a suicidal crisis is not necessarily drawn-out in the same way, which might be one explanation of the lesser burden in our participants than in family members of patients with, for example, schizophrenia.

When it comes to the quality of the relationship with the patient, the participants reported high levels of hostility and criticism in both pre- and post-intervention assessments. Moreover, they expressed notably higher existence of emotional over-involvement than in a clinical sample [37; 26] but similar to the earlier FC studies on BPD parents [38]. High emotional over-involvement means more negative than positive behavior in the interaction between family members. Studies have shown that high $\mathrm{EE}$, which includes emotional over-involvement, is associated with relapse in depression and schizophrenia [39]. However, it has also been shown that higher levels of emotional over-involvement in families with a member diagnosed with BPD, is associated with better clinical outcome and facilitated recovery [39; 40].

Family members of suicidal patients are burdened in their daily life; they feel guilt and perceive additional stress, as noted above. In this study this burden was expressed in worry if own behavior had worsened the situation, in guilt over not having done enough to prevent the suicide attempt or if one in any way had been involved in the process of the suicide attempter's illness. Additionally they felt that they were limited in their own activities, like having to stay home from work. The results from the present study suggest that the DBT-based skills training program may reduce burden in family members of suicidal patients. Contrary to Sin and Norman's conclusion in their review [41] of psychoeducational interventions for family members of people with schizophrenia, the participants' burden in our study was affected in a major way, especially in regards to the worry and guilt they were feeling before entering FC.

The guilt and self-criticism was reduced, as was the worry about the patient's future. Post-intervention assessment clearly showed that the experienced burden was significantly reduced after completion of the program with a medium effect size. This result is concordant with the outcome in other studies of FC and the pilot study of FC for family members of suicidal patients $[7 ; 42 ; 13]$. The intervention includes several factors that could be of importance for this change. Family members are informed of the suicidal process and underlying factors. They are taught new strategies to handle their own emotional responses better 
and they meet others in the same situation. These factors could be assumed to contribute to this improvement.

The results on QAFM showed no significant change in reported distress in the relationship with the patient. The level of strain was high in all subscales in QAFM in both pre and post-intervention assessment. Since results on this scale were significant in the pilot study [13] as well as in other studies on FC, one might wonder why the capacity to accomplish a change in the relationship was low in this study. One consideration is that the dyad is only represented by one part. If the intervention included both parties (i.e. the family member and the patient), there might be a greater possibility for an improvement in the relationship. However, the fact that the participants' burden was significantly reduced might be interpreted as a sign of evolvement in the relationship even if not captured by QAFM. An assumption would be that the relationship had been under pressure for a longer period of time, and to be able to change this pattern in a definite matter, it might take more than a nine-week program with only one of the parties.

The scoring in FFMQ implied less wellbeing at baseline in our sample, since they had lower levels of mindfulness skills than a community sample without experience in meditation [42]. For example, the participants in FC had a much higher degree of judgment which may suggest a critical attitude towards themselves. This score did not change between assessments. Post-assessments in the community sample after 8 weeks of extensive mindfulness training according to the protocol by Segal and colleagues [18] indicated improved abilities to focus and for acceptance. The participants in FC were in comparison still less mindful. One subscale in FFMQ, Acting with Awareness, showed a significant change between pre- and post-intervention assessments. The change between pre- and post-intervention assessment was of the same magnitude as the change in the community sample in Liu's study. This result implied an improvement in being more attentive to the situation. It seemed like the participants assimilated mindfulness skills and that they learned acceptance skills to a greater level. To be able to accept the reality that is at present, is the very foundation for change according to DBT, and the skills training program reflects this position throughout the intervention. The Observing subscale showed a trend to be significant. The results suggest a change towards being more mindful in the sense of being able to observe feelings and thoughts with all senses. Together with the increased ability to act with awareness, the participants might lead their life more consciously and according to their inner values.

Regarding the feasibility, the program was successfully implemented. Significant others seemed to be very motivated to assist in the attempt to find better support and educational material for their fellow peers.

As far as we know, there is little emphasis on taking measures of this kind for family and friends of suicidal patients in clinical care settings. FC introduces new skills and strategies in coping with a stressful life situation. The participant is given an opportunity to learn more about the patient's problems and thereby increase his or her understanding of the patient. Furthermore, the stress coping and 
communication skills taught can be useful not only in relation to the patient but also in other areas of the participant's life.

\section{LIMITATIONS}

Participants came from various parts of Stockholm combining prosperous parts of the inner city as well as more socially burdened parts outside the center of Stockholm. However, ethnic differences can be as important as socioeconomic status in regards of care-giving [44]. Being a convenience sample implies a selection bias. We have broad inclusion criteria and little knowledge of for example the participants own experience of psychiatric illness or educational level. But then again, liberal inclusion criteria, recruiting from a defined area and offering the program in a public psychiatric outpatient clinic could also mean strengthening external validity.

Another limitation was the amount of missing data (33\% of the total sample). Participants did express that they found the intervention useful and a way to measure the feasibility further could be to use indepth qualitative assessments. The main limitation though was the lack of a control group which made it difficult to assess the impact of the program fully. Future studies, preferably randomized controlled studies with larger samples, are needed to generate further evidence.

\section{Conclusions}

In conclusion, even if these results regarding preliminary efficacy should be interpreted cautiously due to the open study design, we found that the intervention had good feasibility. Moreover, the program has promising values in meeting the need of significant others in the difficult situation of having a close relation to someone who is suicidal. The benefit of family members' involvement in patients' care is supported by substantial research. Both the individual and the society have a lot to gain from finding a cost-effective way of improving the contact between health care professionals and family members. The family members' exposed position heightens their own risk for health and psychiatric problems. This in itself justifies a serious attempt to prevent such an unfavorable outcome.

\section{Declarations}

\section{Ethics approval and consent to participate:}

The Regional Ethical Review Board in Stockholm approved of the study protocols (Dnr 2013/840-31/3) and participants in the groups 3-14 gave their written informed consent to participate in the research study and the publication of the results. Regarding the first two groups, the Regional Ethical Review Board approved of their inclusion in the study retrospectively. All participants in the first two groups gave their verbal consent for sharing information of the questionnaires.

\section{Consent for publication}


Not applicable.

\section{Availability of data and material:}

The datasets used and/or analysed during the current study are available from the corresponding author on reasonable request.

\section{Competing interests:}

The authors declare that they have no competing interests.

\section{Funding:}

Grants provided through a regional agreement between Umeå University and Västerbotten County Council (ALF) (VLL-582221) and by grants provided by the Stockholm County Council (ALF) (SLL-20150269)

\section{Authors' contributions:}

All authors have contributed to the fulfilment of the study; JJ and MR designed the study, whereas all authors were involved in the analysis and interpretation of the data. MR wrote the first draft of the manuscript. All authors read, revised and approved of the final manuscript.

\section{Acknowledgements}

The authors would like to thank all participants for sharing their stories and for their engagement in their loved ones.

\section{Abbreviations}

ADHD Attention Deficit Hyperactivity Disorder

BAS Burden Assessment Scale

BPD Borderline personality disorder

DBT Dialectical Behavior Therapy

EE Expressed Emotion

FC Family Connections

FFMQ Five Facet Mindfulness Questionnaire

FPE Family psychoeducation

QAFM Questions About Family Members 


\section{References}

1. Pitman AL, Osborn DPJ, Rantell K, King MB. Bereavement by suicide as a risk factor for suicide attempt: a cross-sectional national UK-wide study of 3432 young bereaved adults. BMJ Open. 2016; doi.10.1136/bmjopen-2015-009948.

2. Van Dongen CJ. Survivors of a family member's suicide: implications for practice. Nurse Pract. Am Journal Primary Health Care. 1991;16:31-39.

3. Bowen DJ, Kreuter M, Spring B, Cofta-Woerpel L, Linnan L, Weiner D, Bakken S, Patrick Kaplan C, Squiers L, Fabrizio C, Fernandez M. How We Design Feasibility Studies. Am J Prev Med. 2009;36:452-457.

4. McFarlane WR. Family Interventions for Schizophrenia and the Psychoses:A Review. Fam Process. 2016;doi:10.1111/famp.12235

5. Grácio J, Gonçalves-Pereira M, Leff J. Key Elements of a Family

6. Intervention for Schizophrenia: A Qualitative Analysis of an RCT. Fam Process. 2016; doi:10.1111/famp.12271

7. Hirvikoski T, Waaler E, Lindström T, Bölte S, Jokinen J. Cognitive behavior therapy-based psychoeducational groups for adults with ADHD and their significant others (PEGASUS): an open clinical feasibility trial. ADHD Atten Def Hyp Disord. 2015; doi:10.1007/s12402-014-0141-2

8. Hoffman PD, Fruzzetti AE, Buteau E, Neiditch E, Penney D, Bruce ML,Hellman F, Struening E. (2005). Family Connections: a program for relatives of persons with borderline personality disorder. Fam Process. 2005;44:217-225.

9. Linehan M. Skills training manual for treating borderline personality disorder. New York, NY: The Guilford Press; 1993.

10. Ekdahl S, Idvall E, Perseius, K-I. Family Skills Training in Dialectical Behaviour Therapy: The Experience of the Significant Others. Arch Psychiatr Nurs. 2014;28:235-241.

11. Valentine SE, Bankoff SM, Poulin RM, Reidler EB, Pantalone DW. The Use of Dialectical Behavior Therapy Skills Training as Stand-Alone Treatment: A Systematic Review of the Treatment Outcome Literature. J Clin Psychol. 2015;71:1-20.

12. Magne-Ingvar $U$, Öjehagen A, Träskman-Bendz $L$. The social network of people who attempt suicide. Acta Psychiatr Scand. 1992;86:153-158.

13. Magne-Ingvar U, Öjehagen A. Significant others of persons with mental health problems: The testing of a questionnaire on the burden of significant others. Nord J Psychiatry, 2005;59: 441-447.

14. Rajalin M, Wickholm-Pethrus L, Hursti T, Jokinen J. (2009). Dialectical Behavior Therapy-Based Skills Training for Family Members of Suicide Attempters. Arch Suicide Res. 2009;13:257-263.

15. Sun F-K, Chiang C-Y, Lin Y-H, Chen T-B. Short-term effects of a suicide education intervention for family caregivers of people who are suicidal. J Clin Nurs. 2012;23:91-102.

16. LeCloux M, Maramaldi P, Thomas K, Wharff E. Family Support and Mental Health Service Use Among Suicidal Adolescents. J Child Fam Stud. 2016; doi:10.1007/s10826-016-0417-6 
17. World Health Organization (WHO) https://www.who.int/mental_health/mhgap/evidence/psychosis/en/ (2014). Accessed December 2016.

18. Kabat-Zinn J. Full catastrophe living: Using the wisdom of your body and mind to face stress, pain and illness. New York, NY: Delta Books; 1990.

19. Segal ZV, Williams JMG, Teasdale JD. Mindfulness-based cognitive psychotherapy for depression: A new approach to preventing relapse. New York, NY: Guilford Press; 2002.

20. Fruzzetti AE, Shenk C, Mosco E, Lowry K. Emotion regulation. In: O'Donohue WT, Fisher JE, Hayes SC, editors. Cognitive behavior therapy: Applying empirically supported techniques in your practice. New York, NY: Wiley. 2003. p.152-159.

21. Gu J, Strauss C, Bond R, Cavanagh K. How do mindfulness-based cognitive therapy and mindfulness-based stress reduction improve mental health and wellbeing? A systematic review and meta-analysis of mediation studies. Clin Psychol Rev. 2014;37:1-12.

22. Linehan M. Cognitive Behavioral Treatment of Borderline Personality Disorder. New York, NY: The Guilford Press; 1993.

23. Jungbauer J, Wittmund B, Dietrich S, Angermeyer MC. The Disregarded Caregivers: Subjective Burden in Spouses of Schizophrenia Patients. Schizophr Bull. 2004;30:665-675.

24. Knudson B, Coyle A. Parents' experiences of caring for sons and daughters with schizophrenia: a qualitative analysis of coping. Eur J Psychother Counsell Health. 2002;5,:169-183.

25. Reinhard SC, Gubman GD, Horwitz AV, Minsky S. Burden Assessment Scale for families of the seriously mentally ill. Eval Program Plann. 1994;17:261-269

26. Ivarsson A, Sidenvall B, Karlsson M. The factor structure of the Burden Assessment Scale and the perceived burden of caregivers for individuals with severe mental disorders. Scand J Caring Sci. 2004;18:396-401.

27. Hansson K, Jarbin H. A new self-rating questionnaire in Swedish for measuring expressed emotion. Nord J Psychiat. 1997;51:287-297.

28. Brown GW, Birley JLT, Wing JK. Influence of family life on the course of schizophrenic disorders: a replication. Br J Psychiatry. 1972;121:241-258.

29. Baer RA, Smith GT, Hopkins J, Krietemeyer J, Toney, L. Using self-report assessment methods to explore facets of mindfulness. Assessment. 2006;13:27-45. SPSS 23.0 (2015). SPSS for Windows ${ }^{\circ}$. Chicago, IL: SPSS Inc.

30. Little RJ, Rubin DB. Statistical analysis with missing data. 2nd ed. New York, NY: John Wiley; 2002.

31. Morris SB, DeShon RP. Combining effect size estimates in meta-analysis with repeated measures and independent-groups designs. Psychol Methods. 2002;7:105-125.

32. Cohen J. Statistical power analysis for the behavioral sciences. 2nd ed. Hillsdale, NJ: Lawrence Earlbaum Associates; 1998. 
33. Fruzzetti AE, Iverson, KM. Mindfulness, Acceptance, Validation, and Individual Psychopathology in Couples. In: Hayes SH, Follette VM, Linehan MM, editors. Mindfulness and Acceptance, expanding the cognitive-behavioral tradition. New York, NY: The Guilford Press. 2004. p.170-191.

34. Hoffman PD, Fruzzetti AE, Swenson CR. Dialectical Behavior Therapy Family Skills Training. Fam Process. 1999; 38:399-414.

35. Drossel C, Fisher JE, Mercer V. A DBT Skills Training Group for Family Caregivers of Persons With Dementia. Behav Ther. 2011;42:109-119.

36. Wilks C, Valenstein-Mah H, Tran H, King AMM, Lungu A, Linehan M. Dialectical behavior Therapy Skills for families of individuals with Behavioral Disorders: Initial feasibility and Outcomes. Cogn Behav Pract. 2017; doi:10.1016/j.cbpra.2016.06.004

37. Lundblad AM, Hansson K. Relational problems and psychiatric symptoms in couple therapy. Int J Soc Welf. 2005;14:256-264.

38. Lundh L-G, Wångby M. Programmet Familjeband som anhörigstöd inom psykiatrin - en svensk utvärdering. Institutionen för psykologi, Lunds universitet; 2007.

39. Hooley JM. Expressed Emotion and Relapse of Psychopathology. Annu Rev Clin Psychol. 2007;3:329-352.

40. Hooley JM, Hoffman PD. Expressed emotion and clinical outcome in borderline personality disorder. Am J Psychiatry. 1999;156:1557-1562.

41. Sin J, Norman I. Psychoeducational Interventions for Family Members of People With Schizophrenia: A Mixed-Method Systematic Review. J Clin Psychiatry. 2013;74:1145-1162.

42. Hoffman,PD, Fruzzetti AE, Buteau E. Understanding and engaging families: An Education, Skills and Support Program for relatives impacted by Borderline Personality Disorder. J Ment Health. 2007;16:69-82.

43. Liu X, Xu W, Wang Y, Williams JMG, Geng Y, Zhang Q, Liu X. Can Inner Peace be Improved by Mindfulness Training: A Randomized Controlled Trial. STRESS HEALTH. 2015;31:245-254.

44. Horwitz AV, Reinhard SC. Ethnic Differences in Caregiving Duties and Burdens Among Parents and Siblings of Persons with Severe Mental Illness. J Health Soc Behav. 1995;36:138-150.

\section{Tables}

Table 1. Content of FC during the nine week program 
Program content of the 9 weekly sessions

Session I: Introduction and psychoeducation

General introduction of the program, psychoeducation consisting of research based information about suicidal behavior, risk- and underlying factors and a description of the suicidal process. Furthermore information about related psychiatric disorders, information about pharmacological and psychological treatments.

Session II: Group presentation

Short presentation of the participant and the individual situation.

Session III: Mindfulness

Introduction of the concepts of mindfulness and validation.

Session IV: Emotion

Information of modern theories of emotion including learning about primary and secondary emotions, communicatory aspects of emotions and relationship and function between cognitions, emotions and behaviors.

Session V: Acceptance

The concept of acceptance is defined and discussed. Identification of goals and values regarding the relationship with the patient.

Session VI: Validation

Continuous work with the concept of validation in relationships.

Session VII: Self-validation and self-invalidation

Focus on self-validation and self-invalidation

Session VIII: Problem management

How to define problems and how to effectively handle them, in collaboration with others or by oneself.

Session IX: Retrospect and outlook

The participants are invited to discuss the content of the program.

Table 2. Participant characteristics in the total sample, $(N=132)$ and in the analyzed sample $(n=86)$ 


\section{$N=132 \quad$ Percent $\% \quad n=86 \quad$ Percent $\%$}

\section{Type of relationship}

$\begin{array}{lcccc}\text { Mother } & 55 & 41.7 & 38 & 44.2 \\ \text { Father } & 25 & 18.9 & 19 & 22.1 \\ \text { Partner (male) } & 22 & 16.7 & 14 & 14.2 \\ \text { Partner (female) } & 9 & 6.8 & 4 & 6.6 \\ \text { Child (son) } & 3 & 2.3 & 3 & 3.5 \\ \text { Child (daughter) } & 2 & 1.5 & 1 & 1.2 \\ \text { Brother } & 4 & 3.0 & 2 & 2.3 \\ \text { Sister } & 6 & 4.5 & 2 & 2.3 \\ \text { Friend } & 2 & 1.5 & 1 & 1.2 \\ \text { Grandmother } & 1 & 0.8 & 1 & 1.2 \\ \text { Stepmother } & 1 & 0.8 & 1 & 1.2 \\ \text { Ex husband } & 2 & 1.5 & - & -\end{array}$

Gender distribution

$\begin{array}{lllll}\text { Women } & 76 & 57.6 & 49 & 57 \\ \text { Men } & 56 & 42.4 & 37 & 43\end{array}$

Living in the same household

$\begin{array}{lcccc}\text { Yes } & 64 & 48.5 & 43 & 50 \\ \text { No } & 54 & 40.9 & 35 & 40 \\ \text { No information } & 14 & 10.6 & 8 & 10\end{array}$

Multiple family members

$\begin{array}{lllll}\text { Yes } & 51 & 38.6 & 37 & 43 \\ \text { No } & 81 & 61.4 & 49 & 57\end{array}$

Gender distribution patient

\begin{tabular}{lcccc} 
Women & 105 & 79.5 & 70 & 81.4 \\
Men & 27 & 20.5 & 16 & 18.6 \\
\hline
\end{tabular}

Table 3. Mean (m), standard deviation (sd), mean difference (Mean diff.), paired samples $t$-test ( $t$ ), $p$-value ( $p$ ) and effect size according to Cohen's $d(d)$ for BAS, FFMQ and QAFM at pre- and post-intervention assessments 


\begin{tabular}{|c|c|c|c|c|c|c|}
\hline \multirow[t]{2}{*}{ Scale } & Pre-intervention & Post-intervention & \multirow[t]{2}{*}{$\begin{array}{c}\text { Mean } \\
\text { diff. }\end{array}$} & \multirow[b]{2}{*}{$t$} & \multirow[b]{2}{*}{$p$} & \multirow[b]{2}{*}{$d$} \\
\hline & $\mathrm{m}(\mathrm{sd})$ & $\mathrm{m}(\mathrm{sd})$ & & & & \\
\hline \multicolumn{7}{|l|}{ BAS $n=86$} \\
\hline BAS total & $26.31(11.3)$ & $23.34(12.1)$ & 2.98 & 3.48 & .001 & 0.38 \\
\hline BAS guilt/anxiety & $1.82(0.70)$ & $1.56(0.74)$ & 0.26 & 4.16 & .000 & 0.44 \\
\hline BAS social stress & $0.92(0.67)$ & $0.82(0.63)$ & 0.10 & 2.27 & .026 & 0.25 \\
\hline BAS disrupted activities & $1.40(0.70)$ & $1.27(0.74)$ & 0.13 & 2.16 & .033 & 0.23 \\
\hline \multicolumn{7}{|l|}{ FFMQ $\quad n=54$} \\
\hline Observing & $23.35(6.79)$ & $24.91(6.09)$ & -0.19 & -1.95 & .056 & -0.27 \\
\hline Describing & $25.85(7.33)$ & $25.43(6.63)$ & -0.06 & 0.78 & .437 & 0.10 \\
\hline Acting with Awareness & $25.30(6.01)$ & $23.43(6.26)$ & 0.22 & 2.86 & .006 & 0.39 \\
\hline Non-judging of Inner Experience & $27.93(6.33)$ & $27.63(6.74)$ & 0.01 & 0.37 & .710 & 0.05 \\
\hline Non-reactivity to Inner Experience & $20.07(3.90)$ & $21.06(5.66)$ & -0.06 & -1.34 & .187 & -0.19 \\
\hline \multicolumn{7}{|l|}{$\mathrm{QAFM} n=81$} \\
\hline PC (perceived criticism) & $2.08(0.76)$ & $2.03(0.74)$ & 0.05 & 0.95 & .345 & 0.11 \\
\hline PEI (perceived emotional involvement) & $2.63(0.53)$ & $2.64(0.60)$ & -0.01 & -0.28 & .776 & 0.03 \\
\hline CC (critical comments) & $2.30(0.78)$ & $2.29(0.74)$ & 0.01 & 0.34 & .738 & 0.04 \\
\hline EOI (emotional overinvolvement) & $3.28(0.77)$ & $3.24(0.75)$ & 0.04 & 0.73 & .470 & 0.08 \\
\hline
\end{tabular}

Note: BAS Burden Assessment Scale; FFMQ Five Facet Mindfulness Questionnaire; QAFM Question about family members

\section{Figures}




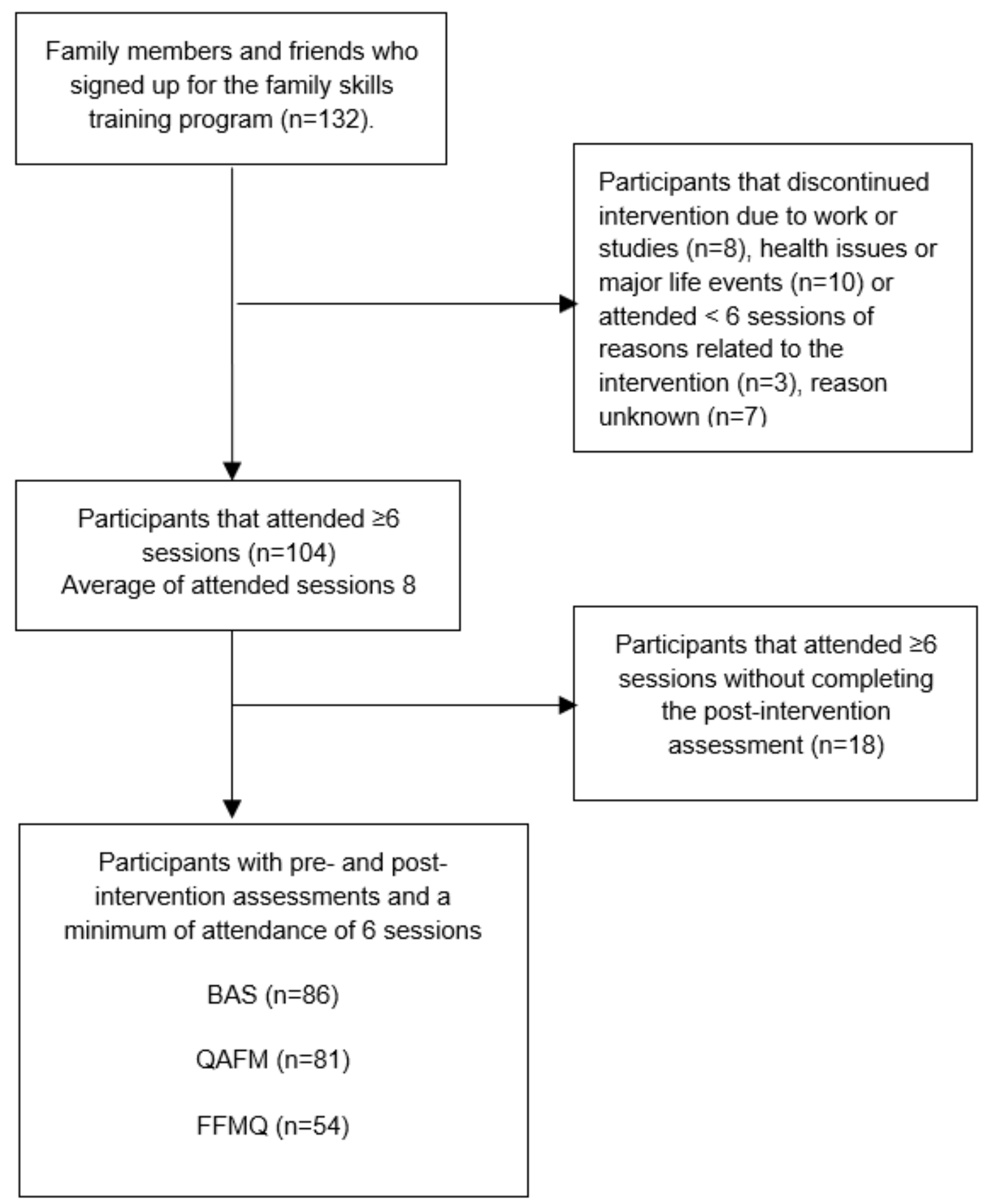

Figure 1

Flowchart of participants 